\title{
Computed tomography-guided radiofrequency ablation combined with transarterial embolization assisted by a three-dimensional visualization ablation planning system for hepatocellular carcinoma in challenging locations: a preliminary study
}

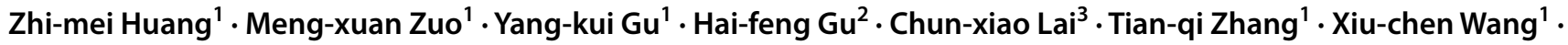 \\ Chao An ${ }^{1}$. J Jin-hua Huang ${ }^{1}$
}

Published online: 31 January 2020

(c) The Author(s) 2020

\begin{abstract}
Objective To assess the clinical efficacy and safety of computed tomography-guided radiofrequency ablation(CT-RFA) combined with transarterial embolization(TAE) assisted by a three-dimensional visualization ablation planning system(3DVAPS) for hepatocellular carcinoma(HCC) in challenging locations.

Methods Data from 62 treatment-naive patients with hepatocellular carcinoma(HCC), with 83 lesions in challenging locations, and who met the Milan criteria and underwent CT-RFA between June 2013 and June 2016 were reviewed. Patients were divided into one of two groups according to different treatment modalities: study group (TAE combined with RFA assisted by 3DVAPS $[n=32]$ ); and control (RFA only $[n=30]$ ). Oncological outcomes included ablation-related complications, local tumor progression (LTP), and overall survival (OS). Univariate and multivariate Cox proportional hazards regression analyses were performed to assess risk factors associated with LTP and OS.

Results HCC lesions (mean size, $1.9 \pm 1.0 \mathrm{~mm}$ in diameter) abutting the gastrointestinal tract $(n=25)$, heart and diaphragm $(n=21)$, major vessels $(n=13)$, and gallbladder $(n=3)$ were treated. A significant difference was detected in LTP between the two groups $(P=0.034)$, with no significant difference in OS between the two groups $(P=0.193)$. There were no severe complications related to ablation. Univariate analysis revealed that sex $(P=0.046)$ and child-turcotte-pugh $(\mathrm{CTP})$ grade $(P<0.001)$ were risk factors for OS, whereas CTP grade and treatment method $(P<0.001)$ were risk factors for LTP. Multivariate analysis revealed that CTP grade B $(P=0.005)$ was independently associated with poor OS, and RFA alone $(P<0.001)$ was independently associated with poor LTP.

Conclusion CT-RFA combined with TAE assisted by a 3DVAPS provided ideal clinical efficiency for HCC in challenging locations and was a highly safe treatment modality.
\end{abstract}

Keywords Computed tomography guided $\cdot$ Transarterial embolization $\cdot$ Radiofrequency ablation $\cdot$ Hepatocellular carcinoma $\cdot$ Challenging locations

Zhi-mei Huang and Meng-xuan Zuo are co-first authors.

Chao An

anchao@sysucc.org.cn

$\triangle$ Jin-hua Huang

huangjh@sysucc.org.cn

1 Department of Minimal Invasive Intervention, State Key Laboratory of Oncology in South China, Collaborative Innovation Center for Cancer Medicine, Sun Yat-sen University Cancer Center, 651, Dongfeng East Road, Guangzhou 510060, People's Republic of China
2 Department of Gynecologic Oncology, State Key Laboratory of Oncology in South China, Collaborative Innovation Center for Cancer Medicine, Sun Yat-sen University Cancer Center, Guangzhou, People's Republic of China

3 Department of Gastroenterology, Huangpu People's Hospital of Zhongshan City, Zhongshan, People's Republic of China 


\section{Introduction}

Hepatocellular carcinoma (HCC) is the sixth most common malignant tumor and the third leading cause of cancer deaths worldwide [1-3]. Surgical management is the standard treatment for early-stage HCC; however, most patients in the intermediate and advanced stages of HCC are not surgical candidates and, are instead, managed with interventional therapies, including local ablative therapy, transarterial chemoembolization (TACE), and transarterial embolization (TAE) [4-6]. Computed tomography (CT)guided radiofrequency ablation (CT-RFA) of liver tumors has the advantages of minimal invasiveness, is repeatable, and is associated with fewer complications and, moreover, has become one of the most important and common clinical treatment methods for HCC in recent years [7-9]. Ablation of intraparenchymal liver tumors generally requires a safety margin of 5-10 mm [10]. However, HCC abutting important organs has been regarded as a "challenging location" and, thus, requires enlarged ablation margins [11]. At the same time, it is easy to cause gastrointestinal, gallbladder, and septal perforation by thermal ablation for $\mathrm{HCC}$ at the above locations. For this reason, there is ongoing controversy regarding thermal ablation of HCC in challenging locations.

TACE or TAE may occlude the main artery supplying the tumor, and subsequent RFA is more effective, reflected by minimized heat loss by convection. Moreover, TACE combined with RFA has demonstrated synergistic cytotoxic effects in HCC, and studies have demonstrated that TACE combined with RFA provides better efficacy than RFA alone $[6,12,13]$. The combined use of TACE or TAE with RFA is also applicable to recurrent or residual HCC. However, TACE increases the risk for liver damage or failure compared with TAE, especially in patients with cirrhosis. Furthermore, RFA alone has a high incomplete ablation rate in HCC lesions abutting high-risk locations including the diaphragm, the gastrointestinal tract, pericardium, gallbladder, and major bile ducts and vascular structures. To our knowledge, few, if any, studies investigating the use of RFA, performed in the area around retained iodized oil immediately following TAE for HCC in challenging locations, have been reported.

It is important to understand the spatial relationship between the tumor and surrounding tissues, especially for thermal ablation of $\mathrm{HCC}$ in challenging locations. A reasonable arrangement of needles and the achievement of an ablative margin are important to the patient's prognosis. A three-dimensional (3D) visualization ablation planning system (3DVAPS) is based on the segmentation and reconstruction of two-dimensional (2D) images (CT or magnetic resonance imaging [MRI]). Multiple ablation spheres of fixed size, covering the target and spatial location relationship between the tumor and surrounding vital organs, can be displayed easily using 3D visualization software. Liu et al. described the clinical application value of 3D visualization in the preoperative treatment planning system in microwave ablation (MWA) for liver cancer, which significantly reduced the local tumor progression (LTP) rate [14].

The present article describes a precise 3D mathematical model. Moreover, oncological outcomes in patients with HCC, who underwent ablation, were compared between a RFA + TAE + 3DVAPS group and an RFA-only group. The purpose of this study was to evaluate the clinical application value of CT-guided RFA combined with TAE assisted by a 3DVAPS to treat HCC in challenging locations.

\section{Materials and methods}

\section{Study design and patient data}

This single-center, retrospective study was approved by the Ethics Committee of the Sun Yat-sen University Cancer Center (Guangzhou, China) and was conducted in accordance with the principles of the Declaration of Helsinki. Electronic medical records of 62 consecutive patients ( 9 female, 53 male; mean $[ \pm \mathrm{SD}]$ age $57.8 \pm 9.2$ years) with 83 lesions, who underwent CT-RFA between June 2013 and June 2016, were reviewed. The diagnosis of HCC was performed according to the guidelines of the European Association for the Study of Liver and the American Association for the Study of Liver Disease. Final diagnosis was confirmed based on histological evidence from needle biopsy specimens. As evaluated by the flow chart presented in Fig. 1, 176 patients were excluded because they did meet the inclusion criteria. Patient inclusion criteria were as follows: (a) single tumor (maximum size $<5 \mathrm{~cm}$, tumor number $<3$ ); (b) sufficient contrast-enhanced imaging (CT or MRI) data before and after MWA; (c) no evidence of extrahepatic metastasis or vascular invasion; and (d) did not undergo hepatectomy or liver transplantation. Patients who received other treatments (e.g., liver resection, liver transplantation, or iodine 125 seed implantation) aside from TAE, those with missing imaging data, individuals with serious medical comorbidities, including heart, lung and renal dysfunction, severe coagulation disorders (e.g., prothrombin time $>25 \mathrm{~s}$, prothrombin activity $<40 \%$, and platelet count $<50$ cells $\times 10^{9} / \mathrm{L}$ ) were excluded from the study.

Before ablation, preoperative planning was performed by three interventional radiologists (J.H.H, Z.M.H and T.Q.Z, with 25,10 , and 10 years' experience with RFA, respectively). Eligible patients were divided into one of two groups according to treatment modality as follows: study group (combined TAE and preoperative planning with the 


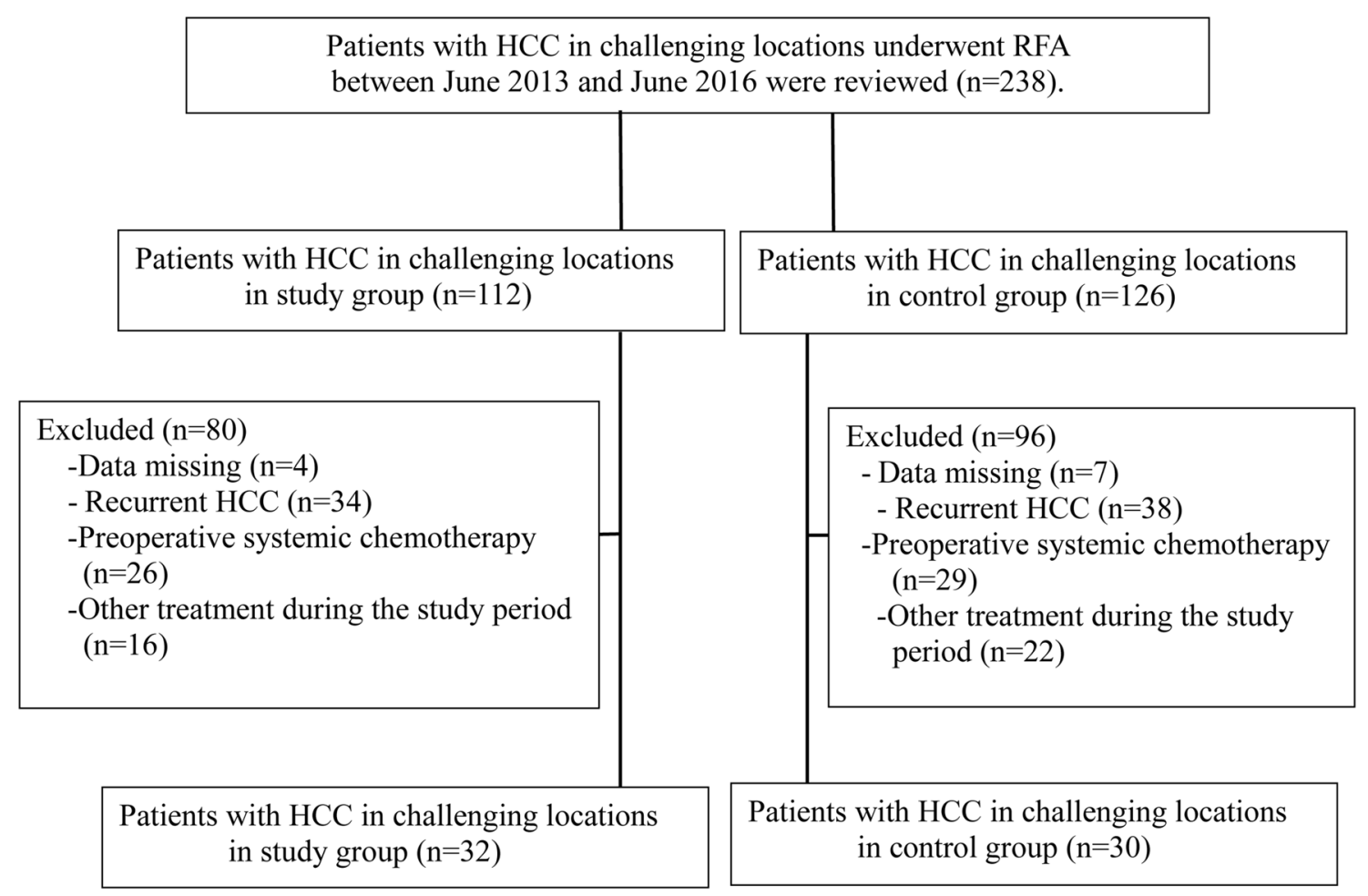

Fig. 1 Flow chart shows patients with HCC in challenging locations enrolled in the study

3DVAPS) and control group (RFA assisted by 2D image preoperative planning).

The following demographic information and clinicopathological data were obtained from each patient: age, sex, comorbidities, hepatitis B surface antigen (HBsAg), childpugh (CTP) grade, maximum tumor diameter, tumor number, liver function-related test examination results (serum albumin, total bilirubin, platelet count, alanine aminotransferase [ALT], and aspartate aminotransferase [AST]). "Challenging location" was defined as a lesion located $<1 \mathrm{~cm}$ (i.e., "abutting") from an important organ, including the stomach, colon, gallbladder, heart, and/or diaphragm, among others. The procedural and/or treatment variables collected included technique effectiveness, postoperative complications, LTP, and date of and status on the last follow-up. The reasons for death were also collected.

\section{D visualization and ablation planning}

A desktop computer (Lenovo, Lenovo Corporation, Quarry Bay, Hong Kong) equipped with an Intel Core i5 (Intel Corporation, Santa Clara, CA, USA), processor for an empirical study in the department was used to perform $3 \mathrm{D}$ visualization operative planning. A series of CT data (slice thickness, $0.625 \mathrm{~mm}$ ) or MRI data (slice thickness, $2.5 \mathrm{~mm}$ ) related to HCC before MWA were converted to DICOM format and then imported into the 3DVAPS (Hokai Company, Zhuhai,
China). The graphical user interface displayed the real-time simulation CT-guided RFA and the 3D visualization planning, as well as the planning path from the transverse, coronal, and sagittal planes. The liver target mass, with a $5 \mathrm{~mm}$ tumor-free margin, vessel and surrounding vital structures, was segmented rapidly (within $2 \mathrm{~min}$ ), was stereo displayed in $3 \mathrm{D}$ visualization. The target sphere volume and quantitative distance between the tumor and surrounding structures was calculated automatically. In the 3D model, puncture path planning was interactive manual simulated according to tumor size, location, the relationship between tumor and surrounding organs, and the preferences of the interventional radiologists. The spherical thermal field was constructed based on the authors' previous experimental studies during preoperative planning. According to tumor size, different permutations and combinations of ablation spheres were adjusted (Fig. 2).

The following principles were complied: for the distance between the tumor boundary and vital structures was larger than $5 \mathrm{~mm}$, expanded ablation was applied, otherwise, conformal ablation was applied; minimizing number of ablation spheres; and minimizing inserting distance to target.

\section{TAE procedures}

TAE was performed with guidance by digital subtraction angiography (Philips, type FD 201250 mA, Amsterdam, 

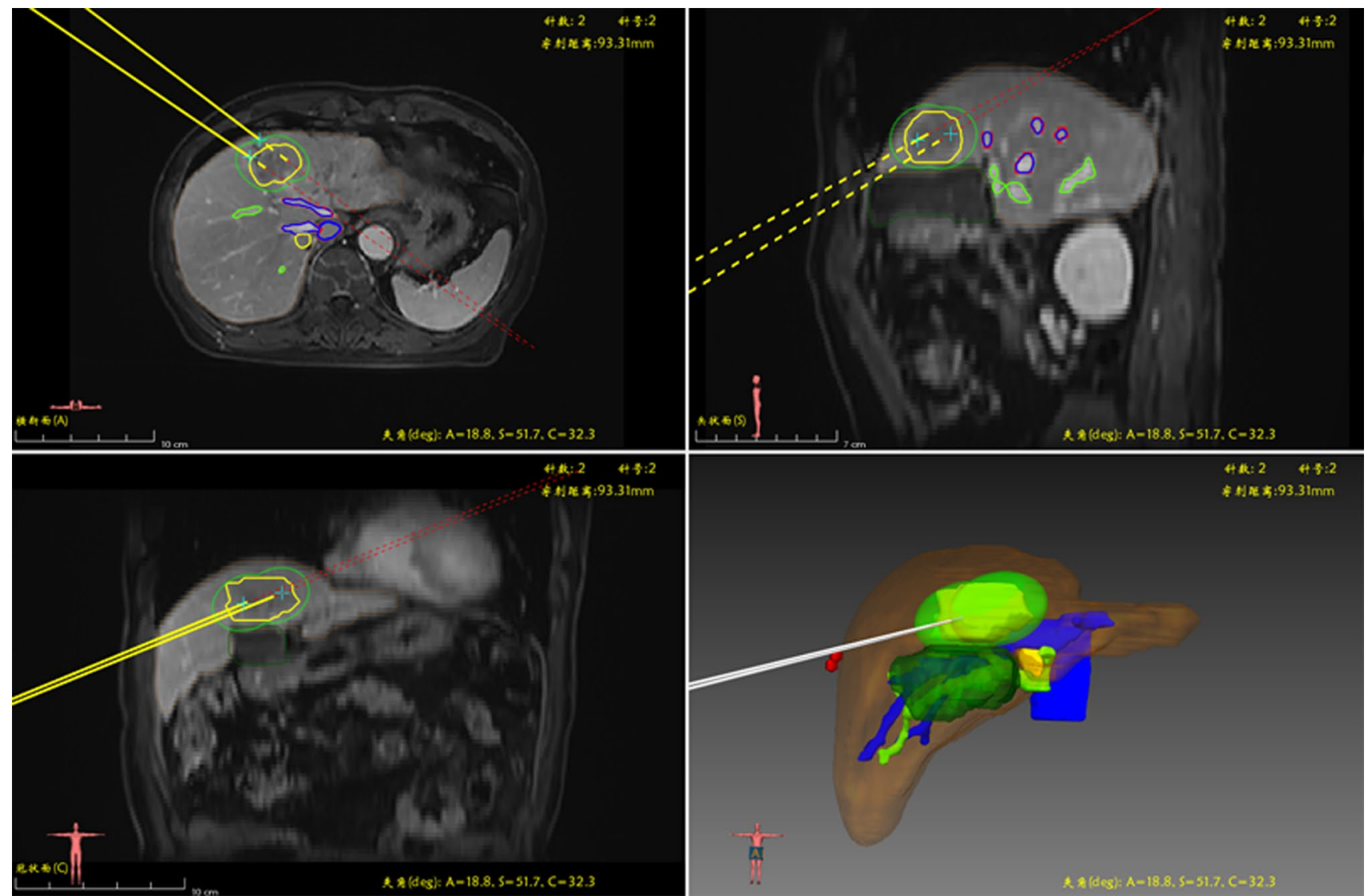

Fig. 2 A 65-year-old female patient with a HCC nodule (3.4 $\mathrm{cm} \times 3.0 \mathrm{~cm} \times 1.9 \mathrm{~cm}$ ) located in $\mathrm{S} 4$ abutting to gallbladder who underwent 3DVAPS. The liver and tumor were segmented rapidly and stereo display by 3DVAPS bases on 2D MRI imaging before

Netherlands). A 5-Fr Yashiro catheter (Terumo, Tokyo, Japan) was introduced into the femoral artery using the Seldinger technique. Angiography of the superior mesenteric artery and common hepatic vessels was performed to assess all tumor vessels. Superselective embolization of the artery directly supplying the tumor was carried out with a microcatheter whenever necessary. The targeting artery was injected by $2-5 \mathrm{~mL}$ iodized oil (Lipiodol; Guerbet, Cedex, France). Therefore, residual or recurred, enhancing targeting tumors were indicated by the iodized oil.

\section{CT-guided RFA procedures}

CT-guided RFA was performed using a 64 multidetectorrow CT (MDCT) scanner (Brilliance CT BigBore; Phillips Medical Systems, The Netherlands). Analgesia was achieved by intravenous administration of remifentanil $(2-3 \mathrm{ng} / \mathrm{ml}$ target concentration infusion) and local injection of 5-15 ml of $1 \%$ lidocaine. Subsequently, the electrode was inserted toward the area of iodized oil retention (Fig. 3). An internally cooled, 17-gauge, $15 \mathrm{~cm}$, single RF electrode, with a 2
RFA, two electrodes were inserted in parallel and the spatial relationship between the simulated three-dimensional thermal field and the gallbladder is clearly visible. Therefore, hydrodissection and thermal monitoring techniques were applied immediately

or $3 \mathrm{~cm}$ long exposed metallic tip (Cool-tip; Covidien, Colorado, MA, USA), was used. The RF energy was delivered in the impedance control mode for 12-16 min, depending on the size of the index tumors. During ablation therapy, the goal was to achieve complete ablation of the iodized oil-marked tumor and to create an ablative margin of at least $0.5 \mathrm{~cm}$ of normal liver parenchyma. When necessary, multiple overlapping applications were performed. Once lesions abutting to important organs were found, hydrodissection and thermal monitoring techniques were applied immediately.

\section{Follow-up}

After ablation, the patients entered the follow-up period, which consisted of contrast-enhanced CT/MRI at 1 and 3 months, and every 3 months thereafter. The follow-up visit also involved several evaluations, including routine physical examination, laboratory tests, including total bilirubin, serum albumin, prothrombin time, and tumor marker levels. Overall survival (OS) and local tumor progression 

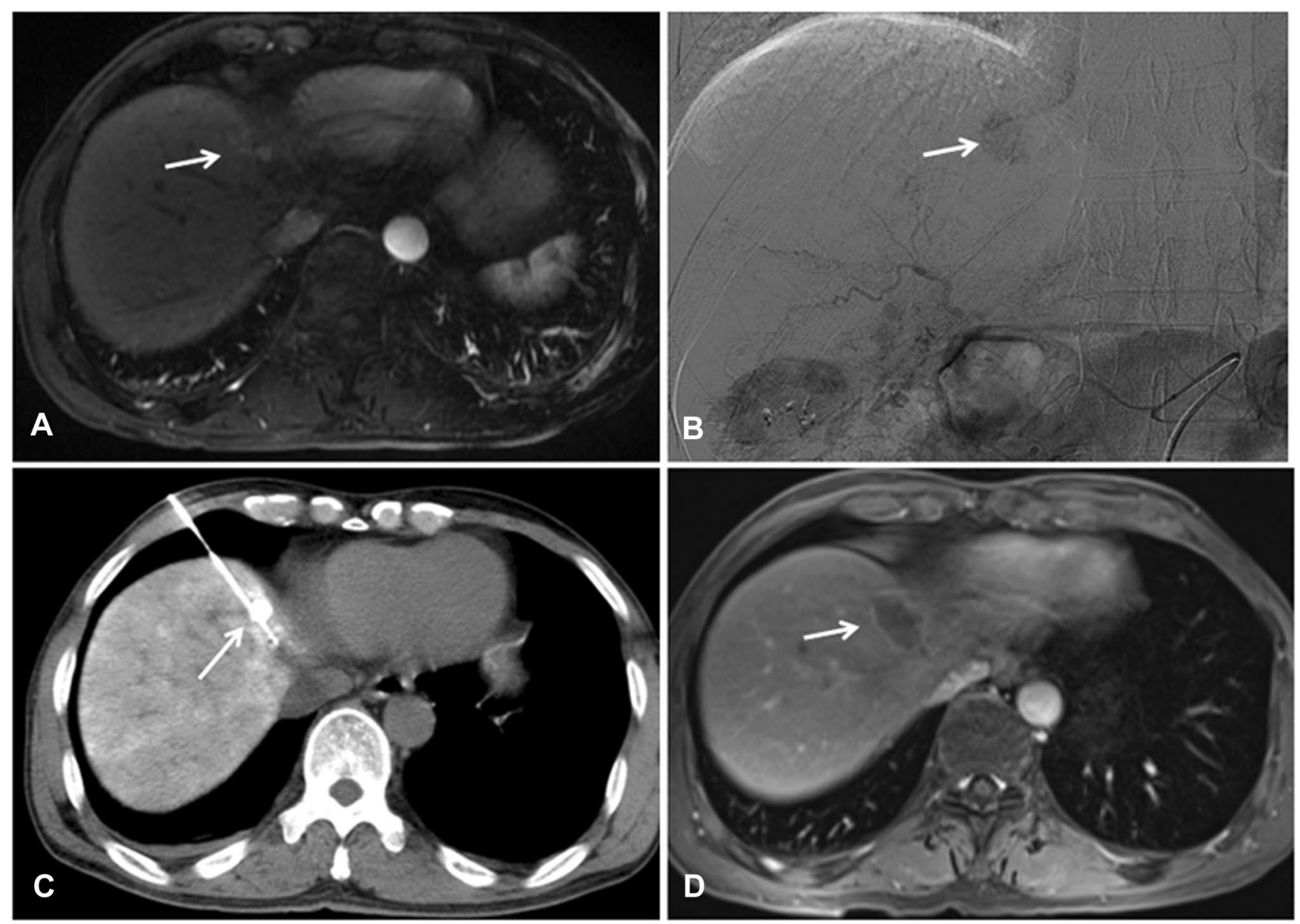

Fig. 3 A 52-year-old male patient was diagnosed with HCC on the basis of scanning by contrast MRI scanning and a high level of serum $\alpha$-fetoprotein(AFP) $51.61 \mathrm{ng} / \mathrm{mL}$, (normal value, $25.0 \mathrm{ng} /$ $\mathrm{mL}$ ). a The HCC nodule (arrow)located in S4 and abutting to heart in MRI scanning images with T2WI high signal; $\mathbf{b}$ the nodule veri-

(LTP) were compared between the study and control groups. OS was defined as the time from the first RFA procedure to the time of death or last follow-up. LTP was defined as the appearance of irregular nodular, scattered, or eccentric patterns of peripheral enhancement around the ablation zone after RFA. Technique effectiveness was defined as the absence of enhancement of any areas of the mass at a follow-up contrast-enhanced imaging examination performed 1 month after RFA. Complications were classified according to the Society of Interventional Radiology Classification system for complications by outcome [15].

\section{Statistical analysis}

Statistical analyses were performed using the Pearson $\chi^{2}$ test, Fisher's exact test, Mann-Whitney $U$ test, or Kruskal-Wallis test, as appropriate. The cumulative rate of local tumor progression and overall survival was estimated using the fied on DSA image (arrow), then a TAE was performed. c A CT exam was performed at 2 days after TAE with iodized oil deposition, then RFA was performed with five insertions under CT guidance (arrow); d completed ablation was verified with no enhancement by MRI image 1 month after ablation (arrow)

Kaplan-Meier method. All statistical tests were 2-sided, and $P<0.05$ was considered to be statistically significant. A Cox proportional hazards model was used to identify the significant effects of risk factors on OS and LTP rate. For all tests, $P<0.05$ was considered to be statistically significant. The analyses were performed using SPSS version 16.0 (IBM Corporation, Chicago, IL, USA) for Windows 3 (CTCAE v3.0, Microsoft Corporation, Redmond, WA, USA).

\section{Results}

\section{Patient and tumor characteristics}

A total of 62 patients with $83 \mathrm{HCCs}$ in challenging locations underwent CT-RFA during the study period. Characteristics of the patients and tumors are summarized in Table 1. The mean age and sex were comparable between the two groups 
Table 1 Baseline characteristics of patients

\begin{tabular}{|c|c|c|c|c|}
\hline Characteristics & Total $(n=62)$ & Study group $(n=32)$ & Control group $(n=30)$ & $P$ value \\
\hline Age $\left(\right.$ years) ${ }^{\mathrm{a}}$ & $57.8 \pm 9.2$ & $56.7 \pm 9.1$ & $59.0 \pm 9.3$ & 0.334 \\
\hline$<65$ & $53(85.5)$ & $27(84.4)$ & $26(86.7)$ & 0.392 \\
\hline$\geq 65$ & $9(14.5)$ & $5(15.6)$ & $4(13.3)$ & 0.846 \\
\hline \multicolumn{5}{|l|}{ Gender } \\
\hline Male & $53(85.5)$ & $26(81.3)$ & $27(90.0)$ & \\
\hline Female & $9(14.5)$ & $6(18.7)$ & $3(10.0)$ & \\
\hline Tumor size $(\mathrm{cm})^{\mathrm{a}}$ & $1.9 \pm 1.0$ & $2.0 \pm 0.8$ & $1.8 \pm 1.2$ & 0.411 \\
\hline$\leq 2$ & $43(69.4)$ & $20(62.5)$ & $23(76.7)$ & \\
\hline $2-3$ & 19 (30.6) & $12(37.5)$ & $7(23.3)$ & \\
\hline Tumor number & 67 & 35 & 32 & 0.260 \\
\hline Single & $58(80.3)$ & $29(76.1)$ & $29(84.2)$ & \\
\hline Multiple & $4(19.7)$ & $3(23.9)$ & $1(15.8)$ & \\
\hline Comorbid diseases & & & & 0.891 \\
\hline Yes & 45 (72.6) & $23(71.9)$ & $22(73.3)$ & \\
\hline No & $17(27.4)$ & $9(29.1)$ & $8(26.7)$ & \\
\hline Cirrhosis & & & & 0.724 \\
\hline Yes & $50(80.6)$ & $26(81.3)$ & $24(80.0)$ & \\
\hline No & $12(19.4)$ & $6(18.7)$ & $6(20.0)$ & \\
\hline HBsAg & & & & 0.724 \\
\hline Yes & $50(80.6)$ & $26(81.3)$ & $24(80.0)$ & \\
\hline No & $12(19.4)$ & $6(18.7)$ & $6(20.0)$ & \\
\hline CTP grade & & & & 0.982 \\
\hline A & $60(95.6)$ & $31(98.9)$ & 29 (92.6) & \\
\hline B & $2(4.4)$ & $1(1.1)$ & $1(7.4)$ & \\
\hline$\alpha$-fetoprotein level $(\mathrm{ng} / \mathrm{ml})^{\mathrm{a}}$ & $14.9(1.4-223.1)$ & $12.4(1.4-66.9)$ & $15.8(2.2-223.1)$ & 0.232 \\
\hline$>20$ & $52(83.9)$ & $28(87.5)$ & $24(80)$ & \\
\hline$\leq 20$ & $10(16.1)$ & $4(12.5)$ & $6(20)$ & \\
\hline Challenging location & & & & 0.538 \\
\hline Abutting gastrointestinal & $25(40.3)$ & $13(40.6)$ & $12(40)$ & \\
\hline Abutting heart and diaphragm & $21(33.9)$ & $11(34.3)$ & $10(33.3)$ & \\
\hline Abutting major vessels & $13(21)$ & $6(18.8)$ & $7(23.3)$ & \\
\hline Abutting gallbladder & $3(4.8)$ & $2(6.3)$ & $1(3.4)$ & \\
\hline \multicolumn{5}{|l|}{ Laboratory examination $^{\mathrm{a}}$} \\
\hline Albumin (g/L) & $36.8 \pm 11.0$ & $37.2 \pm 8.5$ & $36.2 \pm 11.5$ & 0.672 \\
\hline Total bilirubin $(\mu \mathrm{mol} / \mathrm{L})$ & $9.8 \pm 2.3$ & $7.4 \pm 3.2$ & $10.7 \pm 2.6$ & 0.375 \\
\hline Platelet counts $\left(\times 10^{4} / \mathrm{mm}^{3}\right)$ & $145 \pm 23$ & $134 \pm 26$ & $154 \pm 43$ & 0.623 \\
\hline $\operatorname{ALT}(\mu / \mathrm{L})$ & $32.4 \pm 9.4$ & $36.2 \pm 12.4$ & $27.3 \pm 11.1$ & 0.827 \\
\hline $\operatorname{AST}(\mu / \mathrm{L})$ & $26.2 \pm 8.3$ & $31.3 \pm 7.7$ & $34.7 \pm 9.2$ & 0.283 \\
\hline LTP & & & & 0.034 \\
\hline Yes & $16(25.8)$ & $6(18.8)$ & $10(33.3)$ & \\
\hline No & $13(21.0)$ & $26(81.2)$ & $20(66.7)$ & \\
\hline \multicolumn{5}{|l|}{ Metastasis } \\
\hline Yes & $46(74.2)$ & $7(21.9)$ & $6(20.0)$ & 0.698 \\
\hline No & $49(79.0)$ & $25(78.1)$ & $24(80.0)$ & \\
\hline Technique effectiveness & & & & 0.982 \\
\hline Yes & $60(96.8)$ & $31(96.9)$ & $29(96.7)$ & \\
\hline No & $2(3.2)$ & $1(3.1)$ & $1(3.3)$ & \\
\hline Major complications & & & & 1.000 \\
\hline Yes & $0(100)$ & $0(100)$ & $0(100)$ & \\
\hline No & $62(100)$ & $32(100)$ & $30(100)$ & \\
\hline
\end{tabular}

Unless otherwise indicated data are number of patients, with percentage in parentheses

RFA radiofrequency ablation, TACE transcatheter arterial chemoembolization, CTP Child-Turcotte-Pugh, $A L T$ alanine aminotransferase, $A S T$ aspartate aminotransferase

${ }^{\mathrm{a}}$ Data are means standard \pm deviation 
( $P=0.392$ and $P=0.846$, respectively). The mean maximum diameter and tumor number were comparable between the two groups ( $P=0.411$ and $P=0.260$, respectively). Comorbid diseases, cirrhosis, HBsAg, CTP grade and adjacent structures were comparable between the two groups ( $P=0.891, P=0.724, P=0.724, P=0.982$, and $P=0.538$, respectively). $\alpha$-fetoprotein level (AFP), serum albumin levels, total bilirubin and platelet count, alanine aminotransferase (ALT)and aspartate aminotransferase (ALT) were comparable between the two groups $(P=0.232, P=0.672$, $P=0.375, P=0.623, P=0.827$ and $P=0.283$, respectively). LTP in the study group was higher than in control group $(P=0.034)$. Metastasis was comparable between the groups $(P=0.698)$.

\section{Treatment parameters}

Treatment parameters are summarized in Table 2. Thirty patients with 38 tumors underwent a total of 38 treatment sessions in the control group (i.e., RFA only). Thirty-two patients with 45 tumors underwent a total of 45 treatment sessions in the study group (i.e., RFA+TAE+3DVAPS). Thirty-eight tumors were successfully treated in one RFA session in the control group, while 45 tumors were successfully treated in one RFA session in the study group. Ablation time (10.6 $\pm 5.2 \mathrm{~min}$ VS. $13.2 \pm 4.8 \mathrm{~min}, P=0.282)$, ablation power $(147.9 \pm 11.8 \mathrm{~W}$ VS. $143.3 \pm 9.4 \mathrm{~W}, P=0.782)$, ablation energy $(92,128 \pm 3782 \mathrm{~J}$ VS. $88762 \pm 5821 \mathrm{~J}, P=0.172)$ were comparable between the two groups, whereas, puncture times $(4.77 \pm 1.01$ vs. $3.25 \pm 0.42, P=0.047)$ in study group was lower than that in control group. After RFA, complete response $(\mathrm{CR})$ in 27, partial response (PR)in 5 and disease control rate (DCR)was $100 \%$ in study group . Complete response $(\mathrm{CR})$ in 27, partial response (PR)in 1, stable disease in 2 and disease control rate (DCR)was $93.3 \%$ in control group. There were no significant statistical differences were detected in two groups $(P=0.525)$. Estimated blood and loss, cost were comparable between the two groups ( $P=0.214$, and $P=0.184$, respectively) and length of hospital stay in study group was higher than that in control group $(P=0.032)$.

\section{Oncological outcomes after CT-RFA}

The median follow-up period was 24.5 months (range 4.2-58.9 months). Based on follow-up imaging, no significant statistical differences were detected in technique effectiveness rate between the study and control groups $(96.9 \%$ versus $96.7 \% ; P=0.982$ ). The $1-, 2$-, and 3 -year OS rates of the study group and control group were $89.3 \%, 73.5 \%$, $53.7 \%$ and $85.2 \%, 76.4 \%, 59.6 \%$, respectively (Fig. $4 \mathrm{a}$ ), with no significant statistical differences $(P=0.193)$. In contrast, the 1-, 2-, and 3-year LTP rates of the study group and control group were $13.8 \%, 20.6 \%$, and $20.6 \%$ and $31.2 \%, 46.8 \%$, and $58.6 \%$, respectively (Fig. 4b), with differences that were statistically different $(P=0.034)$.

\section{Complications}

Ten of $30(33.3 \%)$ patients in the control group experienced minor complications (minimal liver capsule bleeding $[n=1]$; slight pneumothorax $[n=1]$; and mild pain $[n=8])$. Minor
Table 2 Treatment parameters for $\mathrm{HCC}$

\begin{tabular}{|c|c|c|c|}
\hline Parameters & Study group $(n=32)$ & Control group $(n=30)$ & $P$ value \\
\hline Ablation time $(\mathrm{min})^{\mathrm{a}}$ & $13.2 \pm 4.8$ & $10.6 \pm 5.2$ & 0.282 \\
\hline Ablation power $(\mathrm{W})^{\mathrm{a}}$ & $143.3 \pm 9.4$ & $147.9 \pm 11.8$ & 0.782 \\
\hline Ablation energy $(\mathrm{KJ})^{\mathrm{a}}$ & $88762 \pm 5821$ & $92128 \pm 3782$ & 0.172 \\
\hline Ablation session $^{\mathrm{a}}$ & 1 & 1 & 1.000 \\
\hline Puncture times $(\mathrm{s})^{\mathrm{a}}$ & $3.25 \pm 0.42$ & $4.77 \pm 1.01$ & 0.047 \\
\hline Solid tumor response & & & 0.525 \\
\hline $\mathrm{CR}$ & 27 & 27 & \\
\hline PR & 5 & 1 & \\
\hline SD & 0 & 0 & \\
\hline PD & 0 & 2 & \\
\hline DCR & 100 & 93.3 & 0.281 \\
\hline Estimated blood loss $(\mathrm{ml})^{\mathrm{a}}$ & $8.3 \pm 1.1$ & $7.2 \pm 0.9$ & 0.214 \\
\hline Cost (yuan) $)^{\mathrm{a}}$ & $240874.3 \pm 1105.8$ & $21056.6 \pm 984.2$ & 0.184 \\
\hline Hospital stay $(\mathrm{d})^{\mathrm{a}}$ & $7.8 \pm 3.8$ & $3.2 \pm 0.9$ & 0.032 \\
\hline
\end{tabular}

$H C C$ hepatocellular carcinoma, $T A E$ transcatheter arterial embolization, $R F A$ radiofrequency ablation, $C R$ complete response, $P R$ partial response, $S D$ stable disease, $P D$ progressive disease, $D C R$ disease control rate

${ }^{a}$ Data are means standard \pm deviation 

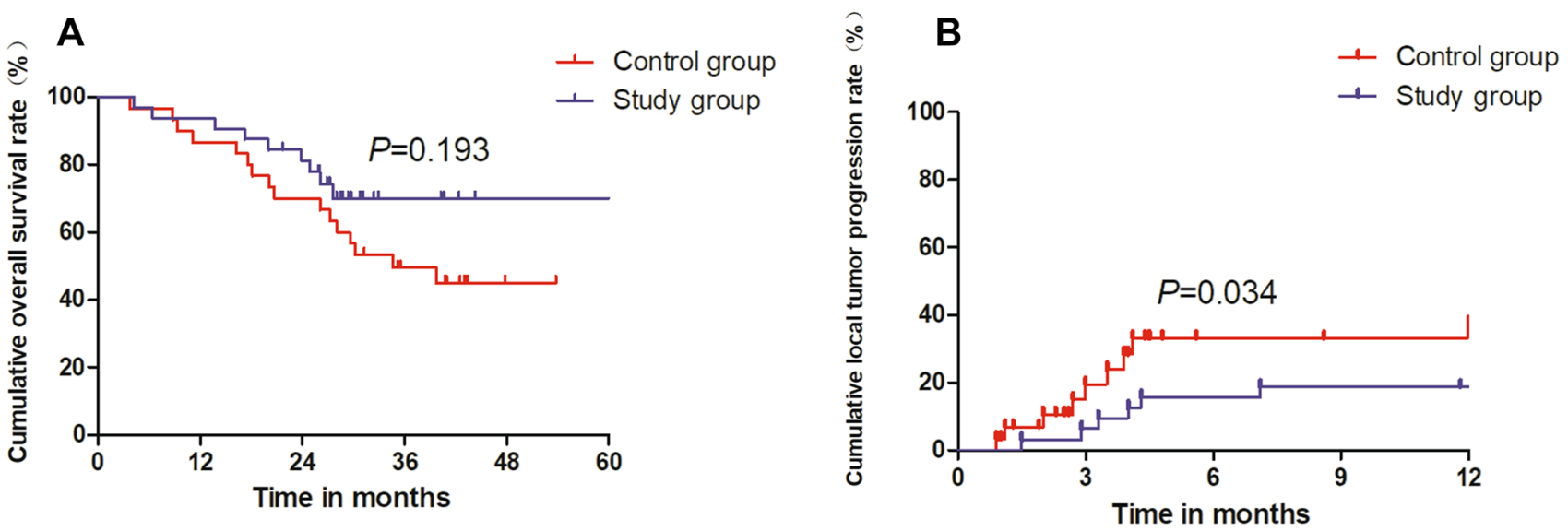

Fig. 4 a graph showed that 1-, 2-, and 3-year OS rates in TAE+RFA group were compared with RFA group $(P=0.193)$; b graph showed that 1-, $2-$, and 3-year LTP rates in TAE+RFA group were lower than RFA group $(P=0.034)$

complications in the study group (11/32 [34.4\%]) included minimal liver capsule bleeding in 3 patients and mild pain in 9. No major complication was observed in either of the two groups and there were comparable between two groups $(P=1.000)$.

\section{Univariate and multivariate analyses}

Univariate and multivariate logistic regression analyses were performed to identify predictors influencing the long-term outcome of patients with HCC in challenging locations who underwent CT-RFA. Univariate analysis revealed statistically significant differences in OS rates, which were dependent on sex (HR 2.954; $P=0.046$ ), and CTP grade (HR 4.534; $P=0.017$ ) (Table 3). Multivariate analysis revealed that CTP grade B was significantly associated with poor OS (HR $2.735 ; P=0.005$ ) (Table 3). Univariate analysis revealed statistically significant differences in LTP rates, depending on CTP grade (HR 3.268; $P=0.018$ ) and treatment method (HR 1.061; $P<0.001)$ (Table 4). Multivariate analysis revealed that RFA alone was significantly associated with poor LTP rate (HR $0.131 ; P<0.001)$ (Table 4).

\section{Discussion}

RFA is generally considered to be a first-line treatment for early-stage HCC, and tumor recurrence due to incomplete ablation is a negative prognostic factor for patient survival $[16,17]$. However, the images used for CT guidance are noncontrasting and cannot precisely identify the tumor focus. Even in contrast CT imaging, the contrast agent does not persist long enough to complete the RFA procedure. Therefore, when RFA is performed under CT guidance, iodized oil retained from previous TACE or TAE procedures is commonly used as a targeting marker, thus improving the complete ablation rate and minimizing the recurrence rate. Numerous studies have confirmed the superior efficacy and safety of TACE or TAE combined with RFA for the treatment of HCC compared with RFA alone, thus significantly improving survival rates $[18,19]$. TAE and RFA are both minimally invasive options that provide the appropriate balance between tumor treatment efficacy and preservation of quality of life. There are several advantages to performing TAE before RFA [20-22]. First, iodized oil can be used to demarcate the range of the tumor(s). Second, because the hepatic artery is the primary source of blood supply to an HCC lesion, occlusion of hepatic arterial flow using TAE can reduce the cooling effect of hepatic blood flow on RFA. Third, the lipiodol used in TAE can improve the likelihood of detection and control of invisible micrometastases in the liver, even when the lesion is solitary and/ or small. Therefore, in the present study, we retrospectively reviewed whether the combination of RFA and previous TAE (1-2 days before) could minimize LTP and increase OS rates.

It is a challenge to perform RFA for HCC in these highrisk locations, including the hepatic dome, subcapsular area, gastrointestinal tract, or gallbladder, among others [23-26], because the ablation volume must be limited to avoid damage to surrounding organs. Therefore, we applied a 3DVAPS to assist with RFA for HCC to achieve multiple ablation spheres of a fixed size covering the target and to observe the spatial location relationship between the tumor and surrounding vital organs more intuitively. The application of 3D visualization technology enables radiologists to perform various manipulations of 3D images, such as free locomotion, scaling and rotation, to produce a puncture plan [27-29]. The system has many distinct characteristics including interactive manual simulation of the insertion number, display 
Table 3 Factors associated with overall survival

\begin{tabular}{|c|c|c|c|c|}
\hline \multirow[t]{2}{*}{ Factors } & \multicolumn{2}{|l|}{ Univariate } & \multicolumn{2}{|l|}{ Multiple regression } \\
\hline & HR $(95 \%$ CI $)$ & $P$ value & HR $(95 \%$ CI $)$ & $P$ value \\
\hline Age (years) & $6.100(4.322,11.878)$ & 0.171 & - & - \\
\hline$<65$ & 53 & & & \\
\hline$\geq 65$ & 9 & & & \\
\hline Gender & $2.954(1.067,4.842)$ & 0.046 & - & - \\
\hline Male & 55 & & & \\
\hline Female & 7 & & & \\
\hline Tumor size (cm) & $2.790(1.478,4.101)$ & 0.731 & - & - \\
\hline$<2$ & 46 & & & \\
\hline $2-3$ & 16 & & & \\
\hline Tumor number & $2.824(1.283,2.364)$ & 0.771 & - & - \\
\hline Single & 44 & & & \\
\hline Multiple & 18 & & & \\
\hline Liver cirrhosis & $3.025(2.071,4.979)$ & 0.273 & - & - \\
\hline Yes & 50 & & & \\
\hline No & 12 & & & \\
\hline Challenging location & $2.154(1.870,6.418)$ & 0.387 & - & - \\
\hline Abutting diaphragm only & 27 & & & \\
\hline Abutting heart and diaphragm & 19 & & & \\
\hline Abutting major vessels & 13 & & & \\
\hline Abutting gallbladder or intestinal canal & 3 & & - & - \\
\hline$\alpha$-fetoprotein level (ng/mL) & $6.642(3.014,11.793)$ & 0.200 & & \\
\hline$\leq 20$ & 30 & & & \\
\hline$>20$ & 32 & & & \\
\hline Child-Pugh grade & $4.534(3.976,5.093)$ & $<0.001$ & $2.735(1.084,6.557)$ & 0.005 \\
\hline A & 60 & & & \\
\hline $\mathrm{B}$ & 2 & & & \\
\hline Treatment & $3.344(2.724,8.965)$ & 0.193 & - & - \\
\hline RFA & 30 & & & \\
\hline TAE+RFA+3DVAPS & 32 & & & \\
\hline
\end{tabular}

Data in parentheses are $95 \%$ confidence intervals

$R F A$ radiofrequency ablation, $T A E$ transcatheter arterial embolization of the virtual thermal field, and calculation of the distance between the target and surrounding vital structures, which can improve ablation success and recurrence outcomes in patients with HCCs in challenging locations.

In the present study, we compared the efficacy and safety between the simultaneous combination of TAE with RFA assisted by 3DVAPS and RFA alone in the treatment of HCC in challenging locations. A significantly higher LTP rate in the RFA group and similar OS rate was found, which was compared with those in the TAE combined with RFA group. These findings suggest that examinations based on contrast imaging underestimate the viable tumor component of the treated lesions, resulting in a lower LTP rate. Koh et al. evaluated the efficacy of CT-guided RFA for recurrent or residual $\mathrm{HCC}$ around the retained iodized oil after TACE in 64 patients with 75 viable HCCs. The authors reported 1- and 2-year LTP rates of $17.5 \%$ and
$37.5 \%$, respectively [30], which was higher than the $13.8 \%$ and $20.6 \%$ LTP rate after TAE combined with RFA in our study. This result appears to be attributed to the important role of preoperative planning in 3DVAPS. Li et al. reported an ultrasound-guided percutaneous MWA technique assisted by a $3 \mathrm{D}$ visualization preoperative treatment planning system and percutaneous transhepatic cholangial drainage with intraductal chilled saline perfusion for larger hepatic hilum HCC, which improved LTP rate and prognosis [28]. In the present study, we identified independent risk factors for OS and LTP in patients with HCC in challenging locations who underwent RFA. Among many risk factors, male sex and poor liver functional reserves (i.e., CTP grade B) were associated with poor OS, and the choice of RFA only was associated with poor LTP. These findings are consistent with previous reports, and further verify the importance of TAE combined with RFA assisted 
Table 4 Factors associated with local tumor progression

\begin{tabular}{|c|c|c|c|c|}
\hline \multirow[t]{2}{*}{ Factors } & \multicolumn{2}{|l|}{ Univariate } & \multicolumn{2}{|l|}{ Multiple regression } \\
\hline & HR $(95 \%$ CI $)$ & $P$ value & $\mathrm{HR}(95 \% \mathrm{CI})$ & $P$ value \\
\hline Age (years) & $4.755(2.994,6.517)$ & 0.415 & - & - \\
\hline$<65$ & 53 & & & \\
\hline$\geq 65$ & 9 & & & \\
\hline Gender & $3.767(2.169,4.365)$ & 0.605 & - & - \\
\hline Male & 55 & & & \\
\hline Female & 7 & & & \\
\hline Tumor size $(\mathrm{cm})$ & $2.464(1.211,3.716)$ & 0.108 & - & - \\
\hline$<2$ & 46 & & & \\
\hline $2-3$ & 16 & & & \\
\hline Tumor number & $2.007(1.534,4.794)$ & 0.676 & - & - \\
\hline Single & 44 & & & \\
\hline Multiple & 18 & & & \\
\hline Liver cirrhosis & $3.038(1.993,6.174)$ & 0.843 & - & - \\
\hline Yes & 50 & & & \\
\hline No & 12 & & & \\
\hline Challenging location & $1.256(0.572,2.112)$ & 0.289 & - & - \\
\hline Abutting diaphragm only & 27 & & & \\
\hline Abutting heart and diaphragm & 19 & & & \\
\hline Abutting major vessels & 13 & & & \\
\hline Abutting gallbladder or intestinal canal & 3 & & - & - \\
\hline$\alpha$-fetoprotein level (ng/mL) & $3.183(2.159,4.207)$ & 0.159 & & \\
\hline$\leq 20$ & 30 & & & \\
\hline$>20$ & 32 & & & \\
\hline Child-Pugh grade & $3.268(2.011,4.525)$ & 0.018 & & \\
\hline A & 60 & & & \\
\hline $\mathrm{B}$ & 2 & & & \\
\hline Treatment & $1.061(0.161,2.870)$ & $<0.001$ & $0.131(0.053,0.322)$ & $<0.001$ \\
\hline RFA & 30 & & & \\
\hline TAE+RFA & 32 & & & \\
\hline
\end{tabular}

Data in parentheses are $95 \%$ confidence intervals

$R F A$ radiofrequency ablation, $T A E$ transcatheter arterial embolization by 3DVAPS in reducing the rate of local progress after local ablation of HCC, especially in challenging locations. In addition, no major complication occurred in the study group. This result suggests that HCC patients who undergo TAE combined with RFA experience a higher quality of life after ablation.

There were several limitations to our study, the first of which was the relatively small patient series; thus, the limited sample size may have reduced statistical power in comparative analyses and, as such, some associations may not have been detected. Second, all of the patients in our cohort were treated at one medical center; thus, referral bias could not be completely avoided. Third, the success of ablation was assessed by radiographic findings versus pathological margin-free status. Therefore, despite intermediate followup being reported, it may take longer for radiographic techniques to detect ablation failures.
In conclusion, results of the current study suggest that a previous TAE (1-2 days before) combined with RFA using retained iodized oil can maximize patient benefits when an initial RFA procedure is performed on a viable tumor. 3D imaging provided more information for controlling LTP. Therefore, the 3DVAPS provides a scientific, objective, quantifiable, and precise strategy for RFA in treating HCC in challenging locations, which could expand its indications.

Acknowledgements This work was supported by the National Science Foundation of China (Grant Number 81371652), 5010 Project of Clinical Research in Sun Yat-sen University (Grant Number 2016002), Science and Technology Program of Guangzhou, China (Grant Number 201704020134).

\section{Compliance with ethical standards}

Conflict of interest The authors declare no conflict of interest. 
Open Access This article is licensed under a Creative Commons Attribution 4.0 International License, which permits use, sharing, adaptation, distribution and reproduction in any medium or format, as long as you give appropriate credit to the original author(s) and the source, provide a link to the Creative Commons licence, and indicate if changes were made. The images or other third party material in this article are included in the article's Creative Commons licence, unless indicated otherwise in a credit line to the material. If material is not included in the article's Creative Commons licence and your intended use is not permitted by statutory regulation or exceeds the permitted use, you will need to obtain permission directly from the copyright holder. To view a copy of this licence, visit http://creativecommons.org/licenses/by/4.0/.

\section{References}

1. Torre LA, Bray F, Siegel RL, Ferlay J, Lortet-Tieulent J, Jemal A. Global cancer statistics, 2012. CA Cancer J Clin 2015;65:87-108.

2. Bray F, Ferlay J, Soerjomataram I, Siegel RL, Torre LA, Jemal A. Global cancer statistics 2018: GLOBOCAN estimates of incidence and mortality worldwide for 36 cancers in 185 countries. CA Cancer J Clin 2018;68:394-424.

3. Forner A, Reig M, Bruix J. Hepatocellular carcinoma. Lancet 2018;391:1301-14.

4. Uhlig J, Sellers CM, Stein SM, Kim HS. Radiofrequency ablation versus surgical resection of hepatocellular carcinoma: contemporary treatment trends and outcomes from the United States National Cancer Database. Eur Radiol 2019;29:2679-89.

5. Pusceddu C, Melis L, Ballicu N, Sotgia B, Melis M, Sanna V, et al. Percutaneous Microwave Ablation Under CT Guidance for Hepatocellular Carcinoma: a Single Institutional Experience. J Gastrointest Cancer 2018;49:295-301.

6. Kim W, Cho SK, Shin SW, Hyun D, Lee MW, Rhim H. Combination therapy of transarterial chemoembolization (TACE) and radiofrequency ablation (RFA) for small hepatocellular carcinoma: comparison with TACE or RFA monotherapy. Abdom Radiol (NY) 2019.

7. Tsukamoto M, Yamashita YI, Imai K, Umezaki N, Yamao T, Kaida T, et al. Long-term Favorable Outcomes of Radiofrequency Ablation for Hepatocellular Carcinoma as an Initial Treatment: A Single-center Experience Over a 10-Year Period. Anticancer Res 2018;38:1047-52.

8. Lee S, Kang TW, Cha DI, Song KD, Lee MW, Rhim H, et al. Radiofrequency ablation vs. surgery for perivascular hepatocellular carcinoma: Propensity score analyses of long-term outcomes. J Hepatol 2018;69:70-8.

9. Francica G, Altiero M, Laccetti E, Pezzullo F, Tanga M, Avitabile G, et al. Long-term follow-up of unresectable medium-large hepatocellular carcinoma nodules treated with radiofrequency ablation using a multiple-electrode switching system. Br J Radiol 2018:20180625.

10. Teng W, Liu KW, Lin CC, Jeng WJ, Chen WT, Sheen IS, et al. Insufficient ablative margin determined by early computed tomography may predict the recurrence of hepatocellular carcinoma after radiofrequency ablation. Liver Cancer 2015;4:26-38.

11. Filippiadis DK, Spiliopoulos S, Konstantos C, Reppas L, Kelekis A, Brountzos E, et al. Computed tomography-guided percutaneous microwave ablation of hepatocellular carcinoma in challenging locations: safety and efficacy of high-power microwave platforms. Int J Hyperthermia 2018;34:863-9.

12. Patidar Y, Garg L, Mukund A, Sarin SK. Early experience of combination therapy of transarterial chemoembolization and radiofrequency ablation for hepatocellular carcinoma measuring $3-7 \mathrm{~cm}$. Indian J Radiol Imaging 2019;29:47-52.

13. Lee JE, Kim JW, Kim HO, Lee BC, Hur YH, Cho SB, et al. Combined Therapy with Transarterial Chemoembolization and Radiofrequency Ablation for Hepatocellular Carcinoma: Does the Degree of Ethiodized Oil Accumulation within the Tumor Affect the Therapeutic Efficacy. J Vasc Interv Radiol 2019;30:370-9.e4.

14. Liu F, Liang P, Yu X, Lu T, Cheng Z, Lei C, et al. A three-dimensional visualisation preoperative treatment planning system in microwave ablation for liver cancer: a preliminary clinical application. Int J Hyperthermia 2013;29:671-7.

15. Duszak R, Mabry MR. Clinical services in interventional radiology: results from the national Medicare database and a Society of Interventional Radiology membership survey. J Vasc Interv Radiol 2003;14:75-81.

16. Sparchez Z, Mocan T, Radu P, Mocan LP, Sparchez M, Leucuta DC, et al. Prognostic Factors after Percutaneous Radiofrequency Ablation in the Treatment of Hepatocellular Carcinoma. Impact of Incomplete Ablation on Recurrence and Overall Survival Rates. J Gastrointestin Liver Dis 2018;27:399-407.

17. Lam VW, Ng KK, Chok KS, Cheung TT, Yuen J, Tung H, et al. Incomplete ablation after radiofrequency ablation of hepatocellular carcinoma: analysis of risk factors and prognostic factors. Ann Surg Oncol 2008;15:782-90.

18. Abdelsalam ME, Murthy R, Avritscher R, Mahvash A, Wallace MJ, Kaseb AO, et al. Minimally invasive image-guided therapies for hepatocellular carcinoma. J Hepatocell Carcinoma 2016;3:55-61.

19. Kim JW, Kim JH, Won HJ, Shin YM, Yoon HK, Sung KB, et al. Hepatocellular carcinomas $2-3 \mathrm{~cm}$ in diameter: transarterial chemoembolization plus radiofrequency ablation vs. radiofrequency ablation alone. Eur J Radiol 2012;81:e189-93.

20. Yamada R, Bassaco B, Dufour L, Collins H, Anderson MB, Hannegan C, et al. Safety and Efficacy of Combined Transarterial Embolization and Percutaneous Radiofrequency Ablation for Liver Tumors Using Cone-Beam CT and Needle Navigation Software in a Single Session. J Vasc Interv Radiol 2019;30:390-5.

21. Yuan Z, Wang Y, Hu C, Gao W, Zheng J, Li W. Efficacy of Percutaneous Thermal Ablation Combined With Transarterial Embolization for Recurrent Hepatocellular Carcinoma After Hepatectomy and a Prognostic Nomogram to Predict Survival. Technol Cancer Res Treat 2018;17:1533033818801362.

22. Liao GS, Yu CY, Shih ML, Chan DC, Liu YC, Yu JC, et al. Radiofrequency ablation after transarterial embolization as therapy for patients with unresectable hepatocellular carcinoma. Eur J Surg Oncol 2008;34:61-6.

23. Hermida M, Cassinotto C, Piron L, Assenat E, Pageaux GP, Escal $\mathrm{L}$, et al. Percutaneous thermal ablation of hepatocellular carcinomas located in the hepatic dome using artificial carbon dioxide pneumothorax: retrospective evaluation of safety and efficacy. Int J Hyperthermia 2018;35:90-6.

24. Liu F, Yu X, Cheng Z, Han Z, Sun Y, Liang P, et al. Comparison of ultrasonography-guided percutaneous microwave ablation for subcapsular and nonsubcapsular hepatocellular carcinoma. Eur J Radiol 2017;91:93-8.

25. Li M, Li Z, Yu X, Liang P, Gao Y, Han Z, et al. Percutaneous radiofrequency ablation of hepatocellular carcinoma adjacent to the gastrointestinal tract. Int J Hyperthermia 2016;32:600-6.

26. Long Y, Yan R, Li K, Luo L, Zeng Q, Tan L, et al. Radiofrequency ablation of liver cancers adjacent to the gallbladder without gallbladder isolation under contrast-enhanced ultrasound monitoring: a preliminary study. Int J Hyperthermia 2019;36:139-45.

27. International BR. Retracted: A Comparison between Three-Dimensional Visualization Guided Hepatectomy and Ultrasonography Guided Radiofrequency Ablation in the Treatment of Small Hepatocellular Carcinoma within the Milan Criteria. Biomed Res Int 2017;2017:8014852.

28. Li X, Yu J, Liang P, Yu X, Cheng Z, Han Z, et al. Ultrasound-guided percutaneous microwave ablation assisted by three-dimensional visualization operative treatment planning system and percutaneous transhepatic cholangial drainage with intraductal chilled saline 
perfusion for larger hepatic hilum hepatocellular ( $D \geq 3 \mathrm{~cm}$ ): preliminary results. Oncotarget 2017;8:79742-9.

29. Liu F, Cheng Z, Han Z, Yu X, Yu M, Liang P. A three-dimensional visualization preoperative treatment planning system for microwave ablation in liver cancer: a simulated experimental study. Abdom Radiol (NY) 2017;42:1788-93.

30. Koh YH, Choi JI, Kim HB, Kim MJ. Computed tomographic-guided radiofrequency ablation of recurrent or residual hepatocellular carcinomas around retained iodized oil after transarterial chemoembolization. Korean J Radiol 2013;14:733-42.

Publisher's Note Springer Nature remains neutral with regard to jurisdictional claims in published maps and institutional affiliations. 